\title{
The importance of different components of normal human serum and lysozyme in the rapid immobilisation of purified Treponema pallidum, Nichols strain
}

\author{
H J H Engelkens, M Kant, P C Onvlee, E Stolz, J J van der Sluis
}

\begin{abstract}
Objectives-To study the role of different components in normal human serum and the role of lysozyme in rapid immobilisation of Percoll purified T pallidum (Nichols).

Materials and methods-The immobilisation of Percoll purified $T$ pallidum was studied after pre-incubations with different serum fractions (Fr) of normal human serum ( Fr 1, containing IgM; Fr 2, containing IgG and a low level of haemolytic complement, and Fr 1 (abs), depleted of IgG). A guinea-pig serum pool was used as a complement source in the immobilisation experiments. The influence was studied of removal of lysozyme from guinea-pig serum on the immobilisation reactions. Further experiments were performed, using a fluorescence technique, to detect C3b depositions on fixed treponemes and treponemes in suspension.
\end{abstract}

Results-Rapid immobilisation of Percoll-purified treponemes by the NHS serum fractions occurred only after preincubation with Fr 1 and Fr 2 simultaneously. This was largely dependent on the presence of a small amount of haemolytic $C$ in $F r$ 2. Removal of lysozyme reduced this rapid rate of immobilisation. In fluorescence experiments it was demonstrated that $\mathbf{C 3 b}$ deposition on fixed (that is damaged) treponemes occurred upon their incubation with Fr 2 or the combination of Fr 1 and 2. However, on treponemes in suspension C3b deposition occurred only after incubation with the combination of Fr 1 and 2.

Conclusion-The rapid immobilisation of Percoll purified treponemes by serum fractions from normal human serum requires antibodies of the IgM and IgG class, together with complement and lysozyme. Omission of one of these reactants slows immobilisation. Our experiments suggest that the reactants act in sequence: the loss of integrity of the outer membrane by an attack by IgM and $C$ offers the opportunity for lysozyme to hydrolyse the peptidoglycan layer surrounding the cytoplasmic membrane of the treponemes, which then is accessible for attack by antibodies and $\mathrm{C}$.

(Genitourin Med 1993;69:280-285)
Introduction

The eradication of Treponema pallidum subspecies pallidum ( $T$ pallidum), the causative organism of syphilis, in patients is often incomplete. This may lead to chronic infection and in some cases to tertiary syphilis. In experimental syphilis in rabbits the persistence of treponemes has also been documented, despite the development of chancre immunity.

The mechanisms of survival of the treponemes for many years in the host are not fully understood. Several hypotheses have been proposed. It may relate to a poor immune response, but experimental results are contradictory. Recently, it was hypothesised that an early down-regulation of the immune response could allow the survival of a small number of treponemes. ${ }^{1}$ Alternatively it was postulated that coverage of the treponemal outer membrane with mucopolysaccharides $^{23}$ or host serum proteins ${ }^{4}$ confers protection against attack by the host's defences upon the treponemes. Although this might explain the presence of pathogenic treponemes together with high titre treponemal antibodies in a host, evidence for the presence of a cover offering protection to the treponemes is lacking. It may be that an absence of antigenicity, due to the presence of only few transmembrane particles in the treponemal outer membrane, may provide a mechanism by which the treponemes evade the host immune response. ${ }^{56}$

In vitro immobilisation of rabbit-derived treponemes by antibodies and complement takes a long time. It was hypothesised that treponemes had first to lose their protective cover before antibodies could gain access to the treponemal surface and complement could be activated to eventually lyse the treponemes. The presence of lysozyme accelerates immobilisation. The location of the substrate of this enzyme has been a subject of discussion. ${ }^{7-9}$ Blanco et al in $1990^{10}$ demonstrated that it was complement-activation and not antibody-binding which was rate limiting in the immobilisation process. The former correlated with the antibody-mediated aggregation of the rare outer membrane protein. Recently, we demonstrated that treponemes, when harvested from rabbit testicles and purified by Percoll centrifugation, were quickly immobilised by normal human serum (NHS). ${ }^{11}$ The immobilisation could be inhibited by fluids from infected and non-infected rabbit testicles. We investigated which com- 
ponents of NHS participate in the rapid immobilisation of purified treponemes.

\section{Materials and methods}

Propagation and extraction of $T$ pallidum. Propagation and extraction of $T$ pallidum (Nichols) were performed as previously described. ${ }^{11}$ Briefly, the testes were minced and $1 \mathrm{ml}$ of serum free basal reduced medium (BRM) without dithiothreitol was added per gram of wet testicular tissue. The mixture was shaken for 45 minutes at room temperature in an atmosphere of $5 \%$ carbon dioxide and $95 \%$ nitrogen, and centrifuged for 10 minutes $(800 \mathrm{~g})$ to sediment gross particulate matter. The fluid layer containing the treponemes was collected and centrifuged at $12000 \mathrm{~g}$ at $4^{\circ} \mathrm{C}$ for 10 minutes to pellet the treponemes. The supernatant was removed and the treponemes were resuspended in fresh BRM and subjected to Percoll (Pharmacia, Uppsala, Sweden) density gradient centrifugation ( $43 \%$ Percoll in BRM) for 30 minutes at $37000 \mathrm{g.}{ }^{12}$ The layer containing the treponemes was collected and used for further experiments.

Enumeration of treponemes. The treponemes were counted using microslides (path length $0.05 \mathrm{~mm}$, Camlab Limited, Cambridge, England, ref: 5005) and the density of treponemes was calculated as previously described. ${ }^{13}$

Human serum pool. A serum pool was prepared from blood samples from 150 blood donors with negative TPHA results. The pool was stored in aliquots at $-70^{\circ} \mathrm{C}$. Portions of $10 \mathrm{ml}$ were used to prepare antibody-containing serum fractions.

Guinea-pig serum pool. Guinea-pigs were bled and their serum was tested individually for the capacity to sustain the viability of Percoll purified treponemes in an assay similar to the immobilisation assay described below. Only those specimens in which treponemal viability was better than $70 \%$ after $22 \mathrm{~h}$ were used to prepare a serum pool. This pool was stored in aliquots at $-70^{\circ} \mathrm{C}$ and was used as the complement source in immobilisation experiments. Samples used in these experiments were thawed only once.

Preparation of serum fractions from normal human serum (NHS). Approximately $10 \mathrm{ml}$ portions from the human serum pool were subjected to Sephadex G-200 gel filtration. The IgM-containing $19 \mathrm{~S}$ and the IgG-containing 7S fractions (designated Fr 1 and Fr 2 respectively) were collected and concentrated to the volume of serum initially applied to the column in Amicon concentration cells equipped with PM10 membranes. After dialysis for $24 \mathrm{~h}$ against Earles balanced salt solution (one change), the fractions were stored in small portions at $-70^{\circ} \mathrm{C}$ until use.

Estimation of complement activity. Complement activity of sera and serum fractions was determined as previously described. ${ }^{14}$

SDS-PAGE and Western blotting. Sodium dodecyl sulphate-polyacrylamide gel elec- trophoresis (SDS-PAGE) and Western blotting, including the blocking and staining procedures of the strips were performed as described previously. ${ }^{11}$ Ten $\mu$ l of Fr 1, Fr 1 (abs) (see below), and human IgG (Nordic, Tilburg, the Netherlands, lot nr. 1-169) as a reference, were appropriately diluted in sample buffer and applied to different slots of the gel after heating in a boiling water bath for four minutes. After completion of the electrophoresis run and the blotting procedure the strips were incubated with conjugate, consisting of the affinity purified gold-labelled IgG fraction from a goat antiserum against heavy and light chains of human IgG (Janssen Life Sciences Products, Beerse, Belgium) for 2 hours, followed by silver enhancement. Separate strips were stained according the Auroprobe staining procedure (Janssen Life Sciences Products) to visualise the polypeptides. The low-molecular weight standards from Pharmacia (Uppsala, Sweden) were used in estimating the size of the visualised polypeptides.

Depletion of IgG. Depletion of IgG from $\mathrm{Fr}$ 1 was accomplished by absorption with Staphylococcus aureus, strain Cowan 1. (Serva, Heidelberg, Germany, lot nr. 16082c). $2.5 \mathrm{ml}$ of Fr 1 was incubated with $0.05 \mathrm{~g}$ dry weight of pre-washed bacteria for $1 \mathrm{~h}$ at $4^{\circ} \mathrm{C}$. The bacteria were pelleted by centrifugation at $27000 \mathrm{~g}$ at $4^{\circ} \mathrm{C}$. The supernatant, designated Fr 1 (abs) was collected and stored in aliquots at $-70^{\circ} \mathrm{C}$. The efficacy of the absorption procedure was controlled by SDSPAGE electrophoresis and immunoblotting.

Estimation of lysozyme. The lysozyme content of guinea-pig serum and bentoniteabsorbed guinea-pig serum, expressed as units $/ \mathrm{ml}$ and of chicken egg white lysozyme (Sigma, St Louis, USA, lot nr. 89F8275), expressed as units/mg, was determined by the lysis of Micrococcus lysodeikticus (Sigma, lot $\mathrm{nr}$. 109F68081) according to the instructions of the supplier. The latter results were used to calculate the amounts of chicken egg white lysozyme to be added to bentonite-absorbed serum to attain the specified number of units finally present in the mixtures for the study of immobilisation.

Depletion of lysozyme. Guinea-pig serum was depleted of lysozyme by absorption with bentonite-SF (Serva, Heidelberg, Germany, cat.nr. 14515) according to Wardlaw. ${ }^{15}$ After completion of the absorption procedure the absorbed serum was checked for its lysozyme and complement contents. Lysozyme could no longer be detected. The complement level had decreased by $25 \%$ as compared with preabsorption levels.

Immobilisation of treponemes. The Percoll purified treponemes were used in a final density of $2 \times 10^{7}$ treponemes $/ \mathrm{ml}$. A sufficient number of treponemes were mixed with $\mathrm{Fr} 1$ or Fr 2 (final content $10 \% \mathrm{v} / \mathrm{v}$ ) or with a $1: 1$ mixture of Fr 1 and Fr 2 (final content $20 \%$ $\mathrm{v} / \mathrm{v})$. These mixtures were supplemented to three-quarters of the final volume with BRM and pre-incubated for 15 minutes at room temperature. Finally, pooled guinea-pig 
serum or bentonite-absorbed pooled guineapig serum was added to a final content of $25 \%(\mathrm{v} / \mathrm{v})$. Aliquots of $0.5 \mathrm{ml}$ of these mixtures were placed in small tubes, which were loosely plugged with cottonwool and incubated in a reduced oxygen atmosphere $(4 \%)$ at $34^{\circ} \mathrm{C} .{ }^{16}$ The percentage of mobile treponemes was determined in wet mounts after $0,1,2,3.5$ and $5.5 \mathrm{~h}$ by observing at least 100 treponemes in randomly selected microscopic darkfields. Control tubes were set up by adjusting a volume containing $4 \times 10^{7}$ treponemes from the various suspensions to 1.5 $\mathrm{ml}$ with BRM. After the pre-incubation period $0.5 \mathrm{ml}$ pooled guinea-pig serum was added. These tubes were treated further as described above. A similar set-up was used to study the capacity of serum from individual guinea-pigs to support the mobility of the treponemes. Here, $4 \times 10^{7}$ treponemes, suspended in $1.5 \mathrm{ml} \mathrm{BRM}$, were pre-incubated and mixed with $0.5 \mathrm{ml}$ of individual guineapig serum. Aliquots of these mixtures were stored and read as described above with additional readings after $22 \mathrm{~h}$.

Fluorescence. Three types of fluorescence experiments were performed to detect $\mathrm{C} 3 \mathrm{~b}$ depositions on treponemes. In the first type $50 \mu \mathrm{l}$ from a Percoll purified treponeme suspension (density $2 \times 10^{7}$ ) was applied to glass slides, which were air-dried and heatfixed. It was demonstrated previously that this procedure damages the treponemal outer membrane. ${ }^{11}$ These slides were overlaid with two drops of Fr 1, Fr 2 or a 1:1 mixture of Fr 1 and Fr 2 for 30 minutes at room temperature. The second type of experiments was performed on treponemes which had been incubated in suspension with $\mathrm{Fr} 1, \mathrm{Fr} 2$ or their 1:1 mixture. In the third type of experiments the treponemes in the tubes with the reaction mixtures for immobilisation were used. In the type 2 and type 3 experiments the contents of the tubes were supplemented

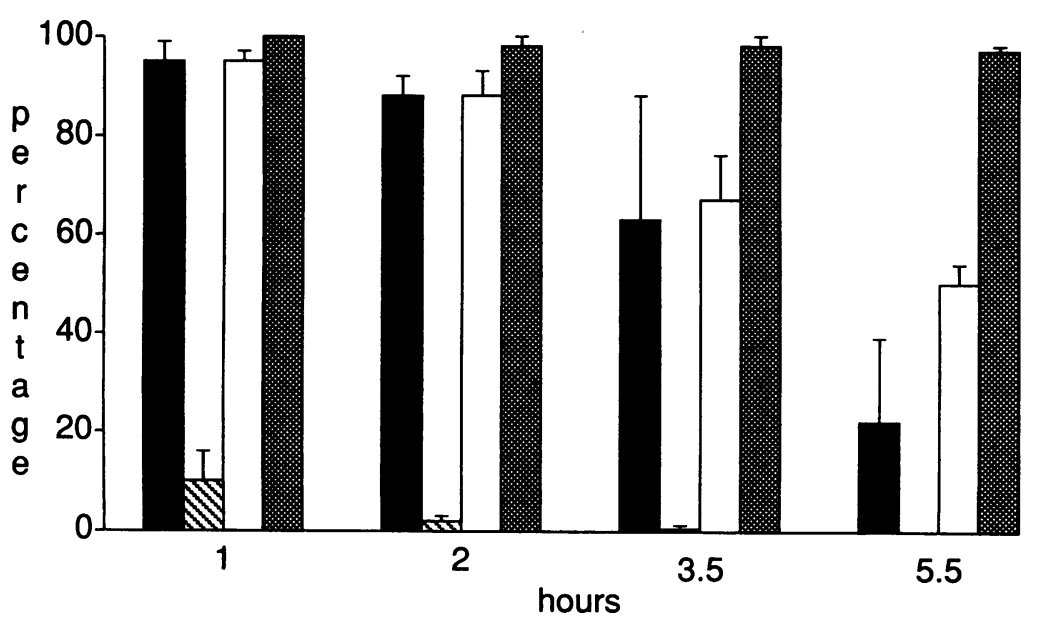

Fr1 Fr $1+2 \square$ Fr2

Figure 1 Immobilisation of Percoll purified treponemes. Treponemal mobility after preincubation with fraction 1, fraction 2, the mixture of fractions 1 and 2, or BRM ( = control). Pooled guinea-pig serum was used as a complement source throughout these experiments. Results are expressed in percentages of mobile treponemes. Means and standard deviations of the results of experiments with six treponemal suspensions originating from different rabbits are shown. with $10 \mathrm{ml} \mathrm{BRM}$ and centrifuged at $12000 \mathrm{~g}$ at $4^{\circ} \mathrm{C}$ for 10 minutes. The supernatant was removed and the pelleted treponemes were resuspended in $1 \mathrm{ml} B R M$. The suspension was pipetted into a Petri dish equipped with a coverglass. After centrifugation at $800 \mathrm{~g}$ for five minutes, the coverglasses were rinsed with BRM. In type 1 and type 2 experiments the treponemes were incubated with two drops of a FITC labelled IgG fraction from a goat anti-human $\mathrm{C} 3 \mathrm{~b}$ antiserum (Centraal Laboratorium van de Bloedtransfusiedienst, Amsterdam), in type 3 experiments the treponemes were incubated with a similar fraction from a goat anti-guinea-pig C3b antiserum (Kirkegaard \& Perry Laboratories, Inc, Gaithersburg, USA) for 30 minutes at room temperature. After rinsing, the slides were covered with a coverglass. The coverglasses with adhering treponemes from the Petri dishes were laid upside down on microscopic slides. The preparations were sealed with nail polish and read within $3 \mathrm{~h}$ as previously described. ${ }^{17}$

\section{Results}

A pre-incubation of Percoll purified treponemes with the IgG-containing $\mathrm{Fr} 2$ resulted, after the addition of guinea-pig serum, in a survival of $88 \%$ of the treponemes after two hours and of $50 \%$ after $5.5 \mathrm{~h}$. In similar experiments with the IgM-containing Fr 1 a mean of $88 \%$ survived after $2 \mathrm{~h}$ and $22 \%$ after $5.5 \mathrm{~h}$. A rapid immobilisation of the treponemes was noted only when the treponemes had been pre-incubated with the combined Fr 1 and Fr 2. Two hours after the addition of guinea-pig serum almost all treponemes had been immobilised (fig 1). In the control tubes, a mean of $97 \%$ of the Percoll purified treponemes survived after $5.5 \mathrm{~h}$.

SDS-PAGE electrophoresis and immunoblotting showed that $\mathrm{Fr} 1$ contained some IgG, which was no longer detectable after absorption of this fraction with Staphylococcus aureus, strain Cowan 1. The immobilisation of the purified treponemes after preincubation with Fr 1(abs) occurred more slowly than the immobilisation after pre-incubation with Fr 1 . After $5.5 \mathrm{~h}, 51 \%$ of the treponemes that had been pre-incubated with Fr 1(abs) survived; after pre-incubation with Fr 1 this was $8 \%$. This indicates a role for IgG in the immobilisation process.

An analysis of the complement content of Fr 1 and Fr 2 showed that only Fr 2 produced a haemolysis of sensitised sheep erythrocytes just above control values, which demonstrated that Fr 2 contained a low level of haemolytic complement. A 1:1 mixture of Fr 1 and Fr 2 did not increase the haemolysis of sensitised erythrocytes, indicating that no separation of complement components had occurred during the preparation of serum fractions.

Percoll purified treponemes which had been pre-fixed onto glass slides showed a deposition of human $\mathrm{C} 3 \mathrm{~b}$ after they had been overlaid with Fr 2 or the combination of Fr 1 
Percentage of differently treated treponemes showing deposition of $C 3 b$ after incubation with Sephadex G-200 separated fractions from NHS and their mixture

(Fr 1: 19S fraction, Fr 2: 7S fraction; int. = intensity of fluorescence)

\begin{tabular}{|c|c|c|c|c|c|}
\hline \multirow[b]{2}{*}{ Antigen } & \multirow{2}{*}{$\begin{array}{l}\text { Fr } 1 \\
\% \text { pos }\end{array}$} & \multicolumn{2}{|l|}{ Fr 2} & \multicolumn{2}{|c|}{$\operatorname{Fr} 1+2$} \\
\hline & & $\%$ pos & int. & $\%$ pos & int. \\
\hline $\begin{array}{l}\text { Fixed treponemes } \\
\text { Treponemes in suspension }\end{array}$ & $\begin{array}{l}0 \\
0\end{array}$ & $\begin{array}{r}100 \\
0\end{array}$ & $\begin{array}{l}1-2 \\
-\end{array}$ & $\begin{array}{l}100 \\
100\end{array}$ & $\begin{array}{l}2-3 \\
1-2\end{array}$ \\
\hline
\end{tabular}

and $\operatorname{Fr} 2$, followed by conjugate. No C3b deposition was observed on pre-fixed treponemes that had been overlaid with Fr 1 and conjugate, emphasising the absence of $\mathrm{C}$ from this fraction and demonstrating the nonreactivity of the conjugate with the treponemes. After incubation of purified treponemes in suspension with each fraction or their combination a deposition of $\mathrm{C} 3 \mathrm{~b}$ was observed only after incubation with the combined Fr 1 and Fr 2 (table). These results show firstly the different reactivities of the serum fractions towards damaged and intact treponemes, and secondly that in addition to Fr 2, the presence of $\mathrm{Fr} 1$ is essential for the deposition of $\mathrm{C} 3 \mathrm{~b}$ on treponemes which had been incubated in suspension.

Parallel with their immobilisation, the $\mathrm{C} 3 \mathrm{~b}$ deposition on the treponemes was studied. All treponemes which had been preincubated with the mixture of Fr 1 and Fr 2 showed a deposition of guinea-pig $\mathrm{C} 3 \mathrm{~b}$ of a $3+$ to $4+$ (strong to very strong) intensity, as early as
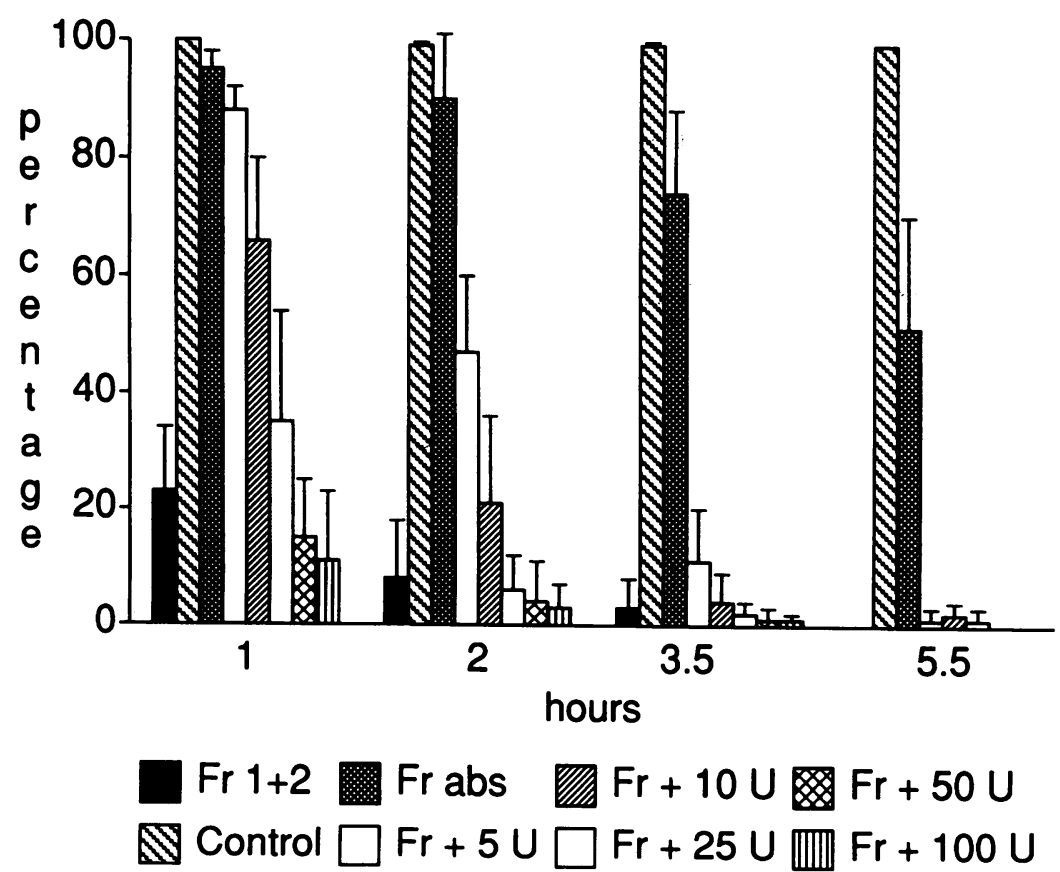

Figure 2 The role of lysozyme in the immobilisation of Percoll purified T pallidum. Treponemal mobility after preincubation with the mixture of fractions 1 and $2(F r 1+2)$,
(or $B R M=$ control), incubated with guinea-pig serum or bentonite-absorbed (Fr abs) guinea-pig serum. Reconstitution of the absorbed guinea-pig serum with lysozyme was performed by addition of graded amounts of chicken egg white lysozyme (from $5 U$ to $100 \mathrm{U})$. Results are expressed in percentages of mobile treponemes. Means and standard deviations of the results of experiments with three treponemal suspensions originating from different rabbits are shown.
$1 \mathrm{~h}$ after incubation. At later times the number of treponemes decreased sharply in the fluorescence preparations. After pre-incubation with $\mathrm{Fr} 1$ or Fr 1(abs) all treponemes showed a very weak fluorescence for $\mathrm{C} 3 \mathrm{~b}$ of a speckled character, which did not change towards the end of the experiments. The same was true of a majority of approximately $60 \%$ of the treponemes that had been preincubated with Fr 2.

It became clear that the rate of immobilisation of the Percoll purified treponemes after pre-incubation with the mixture of $\mathrm{Fr} 1$ and Fr 2 was greatly reduced when Fr 2 was replaced by heat-inactivated Fr 2. Replacement of Fr 1 by heat-inactivated Fr 1 in this mixture hardly influenced the rapidity of the immobilisation as compared with the immobilisation after pre-incubation with the mixture of Fr 1 and Fr 2.

The role of lysozyme is demonstrated in fig 2. As before, the immobilisation of purified treponemes which had been preincubated with the mixture of Fr 1 and Fr 2 proceeded rapidly after the incubation with guinea-pig serum. However, when bentonite-absorbed guinea-pig serum was used the immobilisation proceeded much more slowly: after $5.5 \mathrm{~h}$ $51 \%$ of the treponemes still showed good mobility. The participation of lysozyme in the rapid immobilisation was further demonstrated by the reconstitution of the absorbed guinea-pig serum with graded amounts of chicken egg white lysozyme. In the presence of the lowest amount of lysozyme added (5 U) almost no mobile treponemes were left after $5.5 \mathrm{~h}$ (fig 2). Reconstitution of the absorbed guinea-pig serum with $25 \mathrm{U}$ of lysozyme resulted in an immobilisation as rapid as with unabsorbed guinea-pig serum. Higher amounts of lysozyme did not influence the rate of immobilisation any further. Moreover, these results indicate that the slow immobilisation with bentonite-absorbed guinea-pig serum was indeed due to the depletion of lysozyme and not to a loss of complement from the guinea-pig serum.

To establish a possible reaction sequence of the fractions, pre-incubations were performed with Fr 1(abs) or Fr 2 and after $1 \mathrm{~h}$ of incubation with guinea-pig serum, the missing fraction was added to half of the reaction mixtures. These results are demonstrated in fig 3. In all four types of reaction mixtures the mobility of the treponemes hardly changed during the first hour when one or both fractions were present. At the end of the experiments the immobilisation was greatest in the tubes which had been pre-incubated with Fr 1 (abs) and to which Fr 2 was added later (survival $36 \%$ ). In the tubes which had been pre-incubated with Fr 2, with Fr 1(abs) added later, the survival of the treponemes was $56 \%$. The survival of the treponemes in the tubes containing only one fraction was approximately $70 \%$. In parallel experiments bentonite-absorbed guinea-pig serum was used. Here, in all four types of reaction mixtures approximately $90 \%$ of the treponemes survived after $5 \cdot 5 \mathrm{~h}$. 


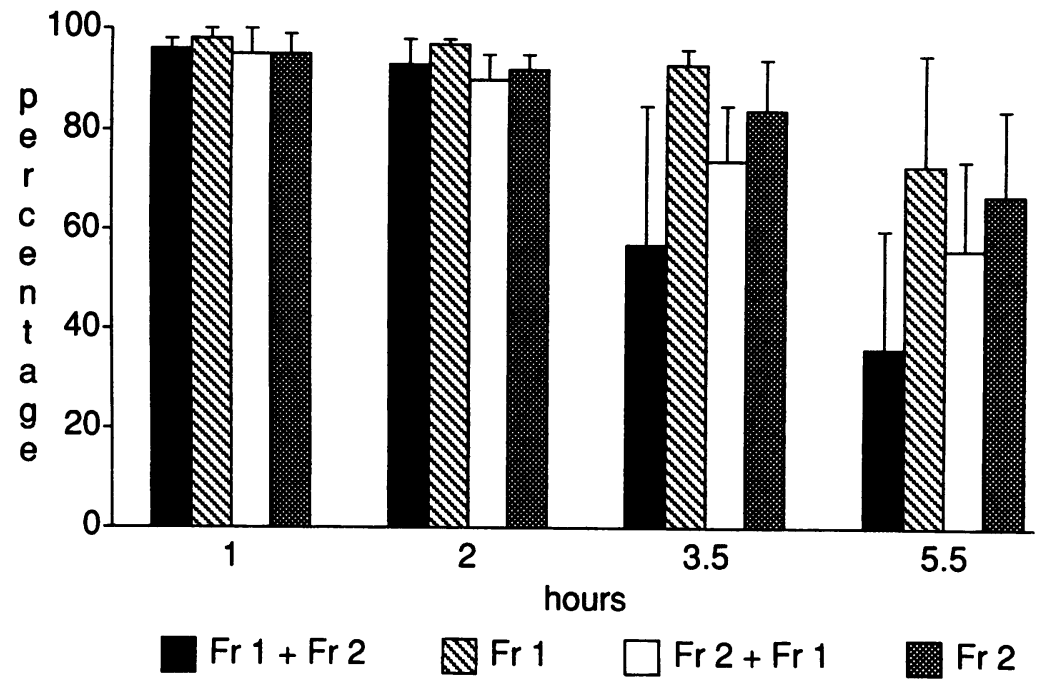

Figure 3 Experiments to study a possible reaction sequence of the fractions. Preincubations were performed with Fr 1(abs) or Fr 2. After one hour of incubation with guinea-pig serum the missing fraction was added to one-half of the reaction mixtures (Fr 1(abs) + Fr 2, or Fr 2 + Fr 1(abs)), or incubation was continued with one fraction only (Fr 1(abs) or Fr 2). Results are expressed in percentages of mobile treponemes. Means and standard deviations of the results of experiments with five treponemal suspensions originating from different rabbits are shown. form antigen-antibody complexes on the treponemal surface, which are capable of C-activation. This may be due to the larger size of this class of antibody molecules, which will allow them to bridge larger distances between epitopes. A preference for the binding of IgM antibodies to adherent treponemes has been demonstrated previously. ${ }^{20}$ This makes it plausible that the first step in the rapid immobilisation of the treponemes is an attack by IgM and $C$. However, the results of the experiments performed with Fr 1(abs) demonstrate that presence of IgM alone does not result in rapid immobilisation. For this to occur IgG is also needed, as emphasised by the rapid immobilisation produced by the mixture of Fr 1 and Fr 2 and the reduction in the immobilisation rate after removal of IgG from Fr 1. The results of the study of the reaction sequence of IgM and IgG antibodies largely agree with this. In this set of experiments more rapid immobilisation was noted when the preincubation was performed with Fr 1 (abs) as compared with a preincubation with Fr 2. These results might point to an inhibition of $\operatorname{IgM}$ by IgG, due to a competition for epitopes on the treponemal surface.

A role of lysozyme in the immobilisation of treponemes was recognised three decades ago. ${ }^{7-9}$ From our experiments it is clear that removal of lysozyme reduces the rate of immobilisation. Reconstitution of the guineapig serum with graded amounts of lysozyme restores the capacity of rapid immobilisation (Fig 2). The results so far obtained demonstrate that rapid immobilisation can be accomplished without presence of lysozyme during the pre-incubation steps. This shows that the action of lysozyme does not occur during the initial steps of immobilisation, but probably only after the outer membrane of the treponemes has been damaged by a complement dependent immunological reaction. This is in agreement with the conclusions of Müller et al. ${ }^{9}$

Our findings demonstrate that the rapid immobilisation of purified treponemes by serum fractions from NHS requires antibodies of the IgM and IgG classes, together with complement and lysozyme. Omission of one of these reactants inhibits the rapid immobilisation. All available evidence indicates that these reactants act in sequence in the rapid immobilisation: the integrity of the outer membrane is first attacked by $\operatorname{IgM}$ and $\mathrm{C}$. The loss of integrity of this membrane provides the opportunity for lysozyme to hydrolyse the peptidoglycan layer surrounding the cytoplasmic membrane of the treponemes. The latter is then accessible for attack by antibodies and C. Presumably IgG plays a major role in this process, since rapid immobilisation without IgG did not occur.
Fr 2" is possible. This does not occur with treponemes in suspension. Here, the IgM containing fraction is needed to accomplish C3b deposition. As previously demonstrated by others, ${ }^{5619}$ the outer membrane of $T$. pallidum shows a scarcity of epitopes. This may be the reason why IgM, but not IgG, can
1 Fitzgerald $\mathrm{TJ}$. The $\mathrm{Th}_{1} / \mathrm{Th}_{2}$-like switch in syphilitic infection: is it detrimental? Infect Immun 1992;60:3475-9. 2 Fitzgerald TJ, Johnson RC. Surface mucopolysaccharides
of Treponema pallidum. Infect Immun 1979;24:244-51. 
3 Zeigler JA, Jones AM, Jones RH, Kubica KM. Demonstration of extracellular material at the surface of pathogenic $T$ pallidum cells. $B r \mathcal{F}$ Venereal Dis 1976; 52:1-8.

4 Alderete JF, Baseman JB. Surface-associated host proteins on virulent Treponema pallidum. Infect Immun 1979; 26:1048-56.

5 Radolf JD, Norgard MV, Schulz WW. Outer membrane ultrastructure explains the limited antigenicity of virulent Treponema pallidum. Proc Natl Acad Sci USA 1989;86:2051-5.

6 Walker EM, Zampighi GA, Blanco DR, Miller JN, Lovett MA. Demonstration of rare protein in the outer membrane of Treponema pallidum subsp. pallidum by freezebrane of Treponema pallidum subsp. pallidum

7 Metzger M, Hardy Jr. PH, Nell EE. Influence of lysozyme upon the treponeme immobilization reaction. $\mathrm{Am} \mathcal{f} \mathrm{Hyg}$ 1961;73:236-44.

8 Kent JF, de Weerdt JB. Enhancement by lysozyme of the sensitivity of Treponema pallidum immobilization tests. Br $\mathcal{F}$ Venereal Dis; 1963;39:37-40.

9 Müller F, Feddersen $H$, Segerling $M$. Studies on the action of lysozyme in immune immobilization of Treponema pallidum (Nichols strain). Immunology 1973; 24:711-9.

10 Blanco DR, Walker EM, Haake DA, Champion CI, Miller JN, Lovett MA. Complement activation limits the rate of in vitro treponemicidal activity and correlates whe rate of in vitro treponemicidal activity and correlates
with antibody-mediated aggregation of Treponema pallidum rare outer membrane protein. $\mathcal{f}$ Immunol 1990;144:1914-21.

11 Engelkens HJH, Kant M, Onvlee PC, Stolz E, van der Sluis J. Rapid in vitro immobilisation of purified Treponema pallidum (Nichols strain), and protection by extraction fluids from rabbit testes. Genitourin Med 1990;66:367-73.

12 Hanff PA, Norris SJ, Lovett MA, Miller JN. Purification of Treponema pallidum, Nichols strain, by Percoll density gradient centrifugation. Sex Transm Dis 1984;11: 275-86.

13 van der Sluis JJ, van Dijk G, Boer M, Stolz E, van Joost Th. Mucopolysaccharides in suspensions of Treponema pallidum extracted from infected rabbit testes. Genitourin Med 1985;61:7-12.

14 Engelkens HJH, Kant M, Onvlee PC, Stolz E, van der Sluis JJ. The influence of different sera on the in vitro immobilisation of Percoll purified Trepone Nichols strain. Genitourin Med 1992;68:20-5.

15 Wardlaw AC. The complement-dependent bacteriolytic activity of normal human serum. I. The effect of $\mathrm{pH}$ and ionic strength and the role of lysozyme. $f$ Exp Med and ionic strength

16 van der Sluis J, Koehorst JAM, Boer AM. Factors that inhibit the adherence of Treponema pallidum (Nichols strain) to a human fibroblastic cell line: development in serum of patients with syphilis. Genitourin Med 1987; 63:71-6.

17 van der Sluis J, van Reede EC, Boer M. Immunoglobulin $G$ subclasses of fluorescent anti-treponema antibodies: evidence for a sequential development of specific antitreponema IgG responses in patients with early syphilis. trepon Microbiol 1986;24:418-23.

18 Frank MM, Fries LF. Chapter 24. Complement. In: Paul WE, editor. Fundamental Immunology. 2nd Ed. New WE, editor. Fundamental Immunolo

19 Cox DL, Chang P, McDowall AW, Radolf JD. The outer membrane, not a coat of host proteins, limits antigenicity of virulent Treponema pallidum. Infect Immun 1992; 60:1076-83

20 van der Sluis JJ, Kant M, Onvlee PC, Stolz E. The inaccessibility of the outer membrane of adherent Treponema pallidum (Nichols strain) to anti-treponemal antibodies, a possible role of serum proteins. Genitourin Med 1990; 66:165-70. 